\title{
Il gergo freddo della democrazia contemplativa: scuola e mass media nel Registro di classe di Sandro Onofri
}

Leonardo Casalino

\section{(2) OpenEdition \\ Journals}

Edizione digitale

URL: http://journals.openedition.org/cei/99

DOI: $10.4000 /$ cei.99

ISSN: 2260-779X

Editore

UGA Éditions/Université Grenoble Alpes

\section{Edizione cartacea}

Data di pubblicazione: 15 juin 2010

Paginazione: 35-39

ISBN: 978-2-84310-168-7

ISSN: 1770-9571

Notizia bibliografica digitale

Leonardo Casalino, «ll gergo freddo della democrazia contemplativa: scuola e mass media nel Registro di classe di Sandro Onofri», Cahiers d'études italiennes [Online], 11 | 2010, online dal 15 décembre 2011, consultato il 28 mars 2021. URL: http://journals.openedition.org/cei/99 ; DOI: https://doi.org/10.4000/ cei.99 


\title{
IL GERGO FREDDO DELLA DEMOCRAZIA CONTEMPLATIVA: SCUOLA E MASS MEDIA NEL REGISTRO DI CLASSE DI SANDRO ONOFRI
}

\author{
Leonardo Casalino \\ Université de Grenoble
}

Il libro di Sandro Onori - Registro di classe - pubblicato dai tipi dell'Einaudi nel 2000, è una opera che si colloca su molteplici confini ${ }^{\mathrm{I}}$. Quello temporale del passaggio da un secolo all'altro con i cambiamenti che lo caratterizzano sul piano dell'insegnamento e dell'educazione pubblica; quello territoriale, una scuola situata in una periferia che fu pasoliniana separata da un altrove (centro città?) in cui il professor Onofri abita; quello politico, segnato dalla fine dell'epoca del militantismo attivo e dalla esplosione della crisi dei partiti tradizionali; quello del sistema dell'informazione e della comunicazione e le sue relazioni contradditorie con il mondo della cultura e dell'istruzione.

Il diario di un anno di scuola è stato ritrovato dalla moglie di Onofri nel disco duro del computer dello scrittore, poco dopo la sua prematura scomparsa. Sin dagli appunti dei primi giorni di scuola si capisce che Onofri era un insegnante appassionato e costantemente impegnato a riflettere sui diversi aspetti della sua professione.

La lotta prima nella quale ogni insegnante deve impegnarsi non è nel far accettare, ma proprio nel non fare rifiutare la lingua dei testi che sottopone ai suoi studenti e che, per il semplice fatto di essere lessicalmente più ricca, retoricamente più varia, sintatticamente più articolata dell'eloquio quotidiano, è automaticamente avvertita come lontana e dunque antidemocratica. Ma la scuola deve impoverire i testi, o deve innalzare il bagaglio linguistico degli studenti? E' una domanda retorica, è ovvio / ma è anche un interrogativo presente in ogni giorno, anzi in ogni ora dell'attività didattica, semplicemente perché sempre più precario si fa il rapporto tra i testi che vi si dovrebbero trattare, e la disponibilità a conoscerli e a penetrarli degli studenti, la

I. Sandro Onofri, Registro di classe, Torino, Einaudi, 2000. 
loro volontà di elevarsi a un livello linguistico necessariamente superiore. La lingua media è diventata un callo intoccabile, lontana dalla spavalderia dei dialetti, permalosa, quasi come il gergo freddo. (Onofri, pp. 23-24.)

Purtroppo tutte le circolari ministeriali ricevute a questo proposito andavano nella direzione di banalizzare e semplificare sempre di più i contenuti delle lezioni e anche l'idea di utilizzare nuovi strumenti - come un concorso di poesie - per stimolare l'uso di un linguaggio più ricco doveva tristemente fare i conti con i pregiudizi che quel "gergo freddo» nascondeva:

E' cosi' oggi si è presentata Enza. Doveva parlarmi privatamente. Della poesia che ha presentato al concorso letterario, ha aggiunto. [...] col viso arrossato per l'imbarazzo ha confessato di botto che quella poesia non era opera sua.

Sono rimasto di stucco. E' vero che sono ormai preparato a tutto: in particolare per le iniziative che esulano dalla stretta attività didattica le sorprese sono all'ordine del giorno. Ma cos'era adesso questa novità? Pure tu, ho detto a Enza, adesso ti metti a fare la furba? Pure tu? Chi l'ha scritta, la poesia? Una cugina più grande? Tua sorella? E Enza, poverina, ha abbassato lo sguardo, e tutto d'un fiato ha confessato che non c'era niente di tutto questo. La poesia era opera di Giovanni, un compagno di classe. Ah, e cos'hai fatto? ho chiesto, sospettoso. Gliel'hai rubata? Macchè professo', ha precisato lei con un sorriso. Me l'ha chiesto lui. E come sarebbe, perché? Perché senno' i compagni, se sanno che lui scrive poesie, cominciano a dirgli che è frocio, professo'. E lui non vuole. Ecco perché. [...]

Pensateci bene, pensate da quale labirinto di pregiudizi emerge questo piccolo, ridicolo problema. (Onofri, 2000, pp. 74-75.)

Un labirinto di pregiudizi che trovava e trova il suo terreno in una società atomizzata, dominata dalla solitudine dei nuclei familiari e da un deserto sociale illuminato dal riflesso delle luci degli schermi televisivi. In cui il dialogo tra generazioni è sempre più complicato e frammentato. Onofri apparteneva a una generazione, quella cresciuta e formata nel corso degli anni Sessanta e Settanta, che sapeva bene che per quanto riguarda il rapporto tra cultura e mass media non esisteva alcun paradiso perduto nel passato o un destino irrimediabile per l'avvenire. Gli scenari futuri dipendevano in realtà anche dalla capacità e volontà di azione politica e sociale degli individui; negli anni Settanta le esperienze positive di Radio Tre diretta da Enzo Forcella e i programmi innovatori alla televisione erano stati il frutto di una lotta per la riforma del servizio radio-televisivo, legata alla stagione dei movimenti collettivi e a un sistema politico costretto ad aprirsi alle novità che emergevano dal conflitto sociale. E tra gli anni Sessanta e Settanta in parallelo alla ricerca politica di un modello nazionale di sviluppo industriale - non legato unicamente alle esigenze dell'economia mondiale - la cultura italiana aveva trovato la 
forza di imporre un proprio immaginario collettivo accanto a quello americano. Le maschere della commedia all'italiana, la variegata ricerca pasoliniana, i romanzi di autori come Bianciardi o Mastronardi avevano saputo raccontare la grande trasformazione della società italiana e i suoi limiti (Crainz, 1996 e 2003).

Questa «democrazia della partecipazione» era stata pero' progressivamente sostituita da una sorta di "democrazia contemplativa» (per riprendere il termine utilizzato da un sociologo russo, Youri Levada, a proposito della Russia di Putin) di cui il diario di Onofri è un intelligente e inquieto registro. Una democrazia in cui un potere politico sempre più personalizzato tende a cancellare tutte quelle istituzioni intermediarie (parlamento, partiti, associazioni, organi costituzionali di controllo) che dovrebbero garantire un corretto rapporto tra chi governa e l'opinione pubblica. In questa prospettiva i media e il loro controllo acquistano una importanza decisiva. Un potere insomma - che senza mettere apertamente in discussione le libertà individuali - spinge però i cittadini verso una generale passività, verso l'accettazione di fatto di uno spettacolo politico che egli mette in scena senza diritto di replica. Questa auto-rappresentazione del potere ha bisogno dei media per essere diffusa e accettata da una società convinta - in nome dell'antipolitica - di poter fare a meno di istituzioni forti e di organizzazioni politiche e sindacali autonome e attive.

Nelle pagine del diario di Onofri si sente l'inquietudine - a cavallo del nuovo secolo - per il destino di una scuola pubblica ogni giorno più subalterna alla logica di un dominio economico in grado di invadere tutti i campi della vita sociale, anche quelli della produzione culturale e dell'arte, capace di integrare in sé tutte le tendenze presenti nella società anche quelle che si propongono riforme più o meno graduali.

Come resistere a questa deriva e come costruire una azione alternativa efficace? Il professore-militante Onofri sapeva resistere individualmente e non senza ottenere qualche risultato, ma per quanto riguarda il secondo aspetto - quello dell'azione collettiva - non poteva che registrare con tristezza e amara ironia la scomparsa, anche all'interno del mondo della scuola, di una figura tipica del Novecento: il militante politico. Dal suo osservatorio della periferia romana, Onofri registrava nel suo diario e cercava di insegnare ai suoi allievi il senso profondo della fine di un secolo, il Novecento, dominato da degli opposti, sempre estremi, sempre assoluti: democrazia e dittatura, ricchezza e miseria, progresso e barbarie, mai capaci di una soluzione stabile, di un equilibrio definitivo (Revelli, 2002). A cominciare da quella che forse ne è stata l'ambivalenza più devastante, il paradosso che ancor oggi ci paralizza: la clamorosa contraddizione tra 
l'onnipotenza dei mezzi tecnici che il secolo ha trovato a propria disposizione - senza dubbio superiore a quella mai raggiunta in ogni altra epoca storica - e la drammatica incapacità da esso dimostrata di raggiungere, senza pagare un prezzo sproporzionato, pressochè tutti i suoi fini. Il dislivello disperante tra l'ossessiva volontà di costruzione del mondo, che ne ha acceso la febbre del fare, e la fragile, incompleta e alla fine dissolta, capacità di controllo sulla distruttività delle proprie macchine e dei propri gesti.

Insegnare per Onofri voleva anche dire riuscire a chiarire a se stesso e ai suoi allievi quale fosse l'eredità che le generazioni precedenti lasciavano a quelle più giovani. E cosa secondo lui di quell'eredità si sarebbe dovuto conservare o cosa si sarebbe dovuto al contrario trasformare o rifiutare. Una eredità pesante e tutta umana. Un mondo, cioè, ormai interamente costruito secondo criteri di efficacia e di razionalità e tuttavia incerto e pericoloso, in cui siamo circondati da pericoli che noi stessi abbiamo prodotto nel tentativo di rendere più sicure le nostre vite. Un universo sociale nel quale la socialità sembra disseccata e dove la stessa ineguaglianza - contro cui la parte migliore del Novecento s'era accanita nel tentativo supremo di ridurla drasticamente, se non di sradicarla - ritorna con dimensioni numeriche scandalose, nelle forme estreme della morte per fame, della migrazione e della distanza incolmabile tra i primi e gli ultimi della terra.

Insomma il registro di Onofri si svolge all'interno di una crisi della politica che è sotto gli occhi di tutti. Da garanzia di ordine e sicurezza, essa si sta rovesciando nel proprio contrario: in fattore di insicurezza, violenza, paura (Revelli, 2003). Lo dimostrano vent'anni circa di nuovo disordine mondiale, dalle cosidette guerre umanitarie, fino all'ultima terribile avventura irachena. Nel corso dell'anno scolastico Onofri cerca di spiegare ai suoi allievi perché si era giunti alla guerra contro la Serbia nel I999 e li invita a comprare dei giornali per informarsi e non essere soltanto dei telespettatori passivi di una crisi così drammatica. Un suggerimento che diventa però l'occasione per constatare, un'ultima volta, quanto fosse diventato difficile lottare contro gli stili di vita e la passività imposti dalla democrazia contemplativa e dal suo gergo freddo:

L'altro giorno, dicevo, c'è stata una specie di spontanea manifestazione contro di me, durante i colloqui. Erano sei signore, minigonne e calze a rete, che si sono sedute, e hanno subito assunto una posa antica. "Quella» posa. Un po' pirandelliana, di colpa e di rimprovero, di un silenzio ammonitore, gli sguardi bassi e ammaccati. Professore, ha cominciato la prima, ma come si fa? Noi siamo lavoratori, non abbiamo mica i beni al sole. Campiamo di stipendio, noi! Noi facciamo mica i professori! Le ho invitate a spiegarsi meglio. Insomma professore, ha ripreso la prima, ma come si fa? Un 
libro al mese! Un libro al mese lei fa comprare ai nostri figli! E noi, poveracci, noi non ce la facciamo più! Allora mi sono difeso, ho detto che mi preoccupo di scegliere solo edizioni tascabili, quindicimila lire al massimo... Ma la sesta, l'ultima, che fremeva, la vedevo che fremeva da quando si era seduta, mi ha interrotto, ha preso a dire sottovoce, scuotendo la testa, sguardo basso e percosso, che adesso si era aggiunta pure la richiesta di comprare i giornali con le pagine sulla guerra. Ci mancava pure la guerra adesso, ha sospirato. Io che mi sentivo ormai una carogna, ho tentato di dire che non c'era bisogno di comprare dei giornali in più, bastava il quotidiano di tutti i giorni. E allora la prima ha alzato una spalla e quale giornale?, ha detto, in casa nostra mica compriamo giornali, ha detto, ci mancherebbe pure questo! Giusto mio marito, la domenica, il Corriere dello Sport. Ma per lei, professore, è sempre domenica. (Onofri, 2000, p. 77.)

\section{Bibliografia}

Crainz Guido, Storia del miracolo economico. Culture, identità, trasformazioni fra anni cinquanta e sessanta, Roma, Donzelli, I996.

Crainz Guido, Il paese mancato. Dal miracolo economico agli anni ottanta, Roma, Donzelli, 2003.

Revelli Marco, Oltre il Novecento, Torino, Einaudi, 200 I.

Revelli Marco, La politica perduta, Torino, Einaudi, 2003.

Onofri Sandro, Registro di classe, Torino, Einaudi, 2000. 
